\title{
The Method of MMC Redundancy Optimization Allocation Based on Multi-objective Optimization
}

\author{
Enshu Jin, Yagu Zhang \\ School of Electrical Engineering, Northeast Electric Power University, Jilin, China
}

\begin{abstract}
When the modular multi-level converter of the sub-module faults, which will cause the unbalanced system, affect the normal operation of the system, so to the MMC arm configuration redundant sub-module is necessary, if the sub-module faults, redundant sub-modules will replace the fault sub-module maintaining the normal operation of system. In this paper, we propose a reasonable and effective method for the configuration optimization of redundant sub-module based on the three indexes, namely, efficient utilization of redundant sub-module, the number of redundant sub-module and the reliability of the system MMC, considering the three factors, establishing a multi-objective optimization function of redundant configuration, By calculating the optimal value to accurately calculate the number of redundant sub-module of MMC, based on the proposed redundancy configuration optimization method, building a simulation model of MMC 5 level in PSCAD, the results verify the rationality and feasibility of the proposed optimization method.
\end{abstract}

\section{INTRODUCTION}

In recent years, in the field of flexible high voltage direct current (HVDC), according to the topology research of modular multilevel converter(MMC) has been widely concerned, modular multilevel converter has the advantages of with low harmonic content, low loss, without a lot of insulated gate bipolar transistor(IGBT) ${ }^{[1-3]}$ Therefore, MMC technology is becoming more and more popular $^{[4]}$, and it will become the mainstream topology in the field of HVDC.

The fault of sub module (SM) is a common fault of $\mathrm{MMC}$, in order to avoid affecting the normal operation of the system, the current protection strategy is commonly used for fault sub module and lock bypass ${ }^{[5-8]}$, The usual practice is to add redundant submodules to the bridge arm and replace the fault sub module with redundant submodules to ensure the security and reliability of the system operation. According to the fault tolerant operation module of the fault, At present, there are two main kinds of redundant submodules, hot standby and cold standby ${ }^{[9]}$, cold standby, when the normal work of the system, the redundant sub module is bypassed, does not participate in the work, When the bridge arm module fails, the fault module is locked, and the redundant sub module is put into operation. The sub module failures to switch to redundant sub modules, which requires a longer time, But the redundant sub module runs exactly the same as that before the failure. Hot standby, redundant sub module can avoid the influence of redundant sub module's charging time and the operation time of power switch on the system, so it is widely applied to engineering. One of the hot standby modes is when a submodule of a bridge arm has a failure, the other bypass bridge arm are the same number of sub modules, To ensure that the system MMC is in a symmetrical running state, a large number of normal sub module is removed, the fluctuation of capacitor voltage is reduced and system reliability increase the sub module; another hot standby mode of operation is only the fault sub module is bypassed, the other Bridge arm remained unchanged, The operation mode is high reliability and easy to operate, and this scheme is more suitable for MMC system.

Redundant sub modules have the more the configuration, the system has the higher the reliability, but the cost also has the higher. The redundant sub modules may involve the problem of low utilization rate; the less configuration, the lower the cost, but the reliability of the system will be reduced ${ }^{[9]}$.

Based on the above analysis, In this paper, the quantitative analysis is made from the following three aspects: the number of redundant sub modules, the effective utilization of redundant modules and the reliability of system MMC, a modular multilevel multiobjective optimization redundancy configuration scheme is proposed, in practical application, it is difficult to the hardware reliability and cost trade-off system weights quantitative relation, Therefore, this paper uses the partition priority to solve the redundant configuration problem of multi-objective optimization. The 5 level MMC model is built through the simulation platform of PSCAD, and the feasibility and effectiveness of the scheme are verified. On the premise of guaranteeing the reliability of the system, the scheme can reasonably configure the number of redundant submodules ${ }^{[10]}$. 


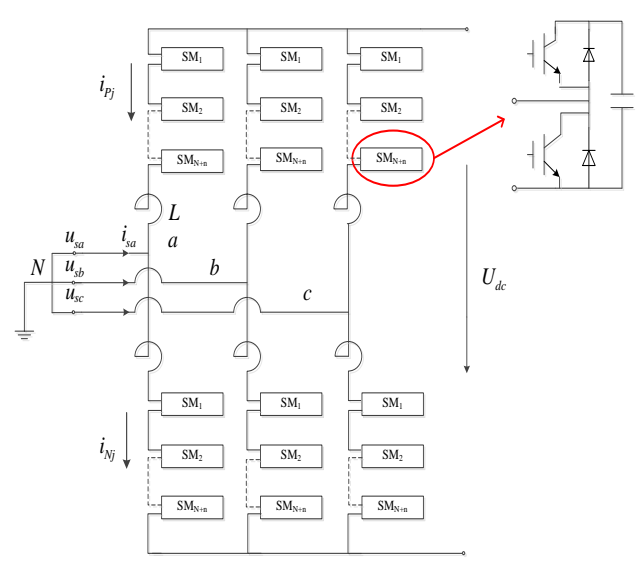

Figure 1: Topological structure of MMC
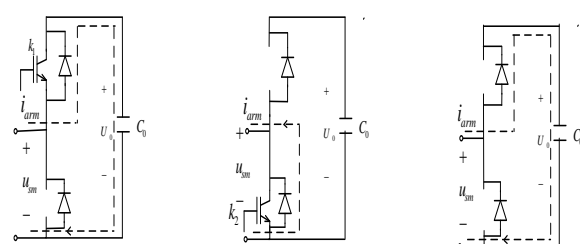

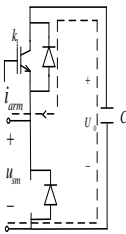

Input

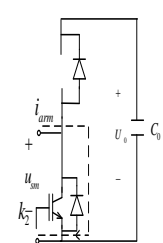

Cut out

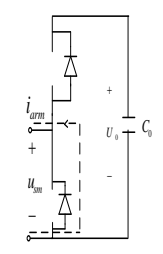

Atresia
Figure 2: Sub-module operation state

\section{Configuration METHOD OF REDUNDANT MODULE}

\subsection{Set up AN Optimal Function of Redundant Configuration}

The sub module fault, in order to ensure the normal operation of the system, you need to configure the redundant sub module, the more the number of redundant sub modules is configured, the greater the reliability of the system, but the effective use of redundant sub modules of the rate will be reduced, the hardware cost will also be increased $^{[17]}$. So it is very important to configure the number of redundant submodules reasonably and effectively. The modular redundancy allocation method proposed in this paper is mainly based on the three indexes of redundant sub modules, the effective utilization of redundant sub modules and the reliability of the system, and establishes the multi-objective function of redundant modules.

\section{Reliability analysis of the system}

The reliability of the system is defined as a period of time $T$ in the normal work of the system of continuous probability, SM is composed of a capacitor, two IGBT and two diodes, its reliability is determined by the reliability of capacitor and diode (for the sake of simplicity, for the reliability of SM, only the reliability of IGBT, diode and capacitor is considered, and other devices are not considered for the time being), the definition of reliability of capacitor is $r_{1}$, the reliability of IGBT is $r_{D}$, the reliability of diode is $r_{C}$, The reliability $r$ of SM is available:

$$
r=r_{1}^{2} r_{D}^{2} r_{C}
$$

MMC is made up of SM, so the reliability of the MMC depends on the reliability of the SM. For MMC of the configuring redundant submodule, if SM fails, the SM will be replaced by redundant SM, so MMC can continue to work normally, then the reliability of MMC system with a redundant sub module is as follows:

$$
R=\left(\sum_{i=0}^{n} C_{N+n}^{i} r^{N+n-i}(1-r)^{i}\right)^{6}
$$

Among them $C_{N+n}^{i}=\frac{(N+n) !}{i !(N+n-i) !}, i$ is the number of fault SM for a bridge arm in MMC, Obviously $i \leq n$

Effective utilization of redundant submodules

The effective number of redundant submodules of MMC is established $H$. According to the calculation idea of MMC system reliability, the number of effective redundant submodules of the MMC system is as follows:

$$
H=6 \sum_{i=0}^{n} i\left[C_{N+n}^{i} r^{N+n-i}(1-r)^{i}\right]
$$

The effective utilization $U$ of redundant submodules is as follows:

$$
U=\frac{H}{6 n}=\frac{1}{n} \sum_{i=0}^{n} i\left[C_{N+n}^{i} r^{N+n-i}(1-r)^{i}\right]
$$

(4)

\section{The number of redundant submodules}

In order to reduce the cost of hardware, the number of redundant sub modules is reduced as much as possible to meet the reliability of the MMC system, that is:

$$
G(n)=\min (n)
$$

In order to make the multi-objective optimization problem easy to calculate and carry on the normalization treatment, there are:

$$
G(n)=\max \left(\frac{N-n}{N}\right)
$$

The redundant configuration multi-objective optimization function is established by establishing redundant sub module effective utilization rate, redundant sub module number and system MMC reliability.

$$
\left\{\begin{array}{l}
R(n)=\max \left\{\left(\sum_{i=0}^{n} C_{N+n}^{i} r^{N+n-i}(1-r)^{i}\right)^{6}\right\} \\
U(n)=\max \left\{\frac{1}{n} \sum_{i=0}^{n} i\left[C_{N+n}^{i} r^{N+n-i}(1-r)^{i}\right]\right\} \\
G(n)=\max \left(\frac{N-n}{N}\right)
\end{array}\right.
$$


In practical application, It is sometimes difficult to weigh the quantitative weight relationship between reliability of system and cost of hardware, and on the premise of guaranteeing the reliability of the system, as far as possible to improve the efficient utilization of redundant submodules and reduce the number of redundant sub modules, therefore, this paper uses the partition priority to solve the redundant configuration problem of multi-objective optimization.

\subsection{Calculation Sstep}

(I) firstly, the effective utilization of redundant submodules, the number of redundant submodules, and the reliability of the system MMC are three ideal targets to determine the expected values, which are transformed into realistic targets, In order to find the potential as much as possible, the expected value is as high as possible in order to find the potential as much as possible for the ideal goal, that is:

$$
\left\{\begin{array}{l}
R(n) \geq R_{\min } \\
U(n) \geq U_{\text {min }} \\
G(n) \geq G_{\min }
\end{array}\right.
$$

(II) add positive and negative deviations for each realistic target, and convert it into the target programming format, that is:

$$
\left\{\begin{array}{l}
\left(\sum_{i=0}^{n} C_{N+n}^{i} r^{N+n-i}(1-r)^{i}\right)^{6}+\eta_{1}-\rho_{1}=R_{\min } \\
\frac{1}{n} \sum_{i=0}^{n} i\left[C_{N+n}^{i} r^{N+n-i}(1-r)^{i}\right]+\eta_{2}-\rho_{2}=U_{\min } \\
\frac{N-n}{N}+\eta_{3}-\rho_{3}=G_{\min }
\end{array}\right.
$$

(III) prioritization: in practical engineering, we should improve the effective utilization of redundant sub modules and reduce the number of redundant sub modules as much as possible while ensuring the reliability of the system. This paper can be divided into 2 priorities, which are divided into the following:

(1) the reliability of the system is the first priority;

(2) the effective utilization of redundant submodules and the number of redundant submodules are second priority.

(IV) establishment of the reached function

The first and second priorities need to minimize negative deviations, that is, $\eta_{1}$ and $\left(\eta_{2}+\eta_{3}\right)$. So the function is as follows:

$$
\text { lex } \min a=\left\{\eta_{1},\left(\eta_{2}+\eta_{3}\right)\right\}
$$

In this paper, the multi-objective programming model of redundant configuration is as follows:

$$
\text { lex } \min a=\left\{\eta_{1},\left(\eta_{2}+\eta_{3}\right)\right\}
$$

$$
\left\{\begin{array}{l}
\left(\sum_{i=0}^{n} C_{N+n}^{i} r^{N+n-i}(1-r)^{i}\right)^{6}+\eta_{1}-\rho_{1}=R_{\min } \\
\frac{1}{n} \sum_{i=0}^{n} i\left[C_{N+n}^{i} r^{N+n-i}(1-r)^{i}\right]+\eta_{2}-\rho_{2}=U_{\min } \\
\frac{N-n}{N}+\eta_{3}-\rho_{3}=G_{\min } \\
n, \rho_{1}, \rho_{2}, \rho_{3}, \eta_{1}, \eta_{2}, \eta_{3} \geq 0
\end{array}\right.
$$

Among $\rho$ the negative deviation, $\eta$ is the positive deviation, $N$ is the number of the normal operating submodules of the system MMC. The reliability $r$ of the $\mathrm{SM}$ is bound to be certain for the engineering practice. $R_{\min } 、 U_{\min } 、 G_{\min }$ is the expected value of an ideal objective function, as high as possible. There is a minimum of the reached function, the number of redundant submodules corresponding to their minimum values is the number of optimal redundant sub modules.

The flow chart of the optimal redundant configuration calculation method based on multi objective optimization is shown in Figure 3.

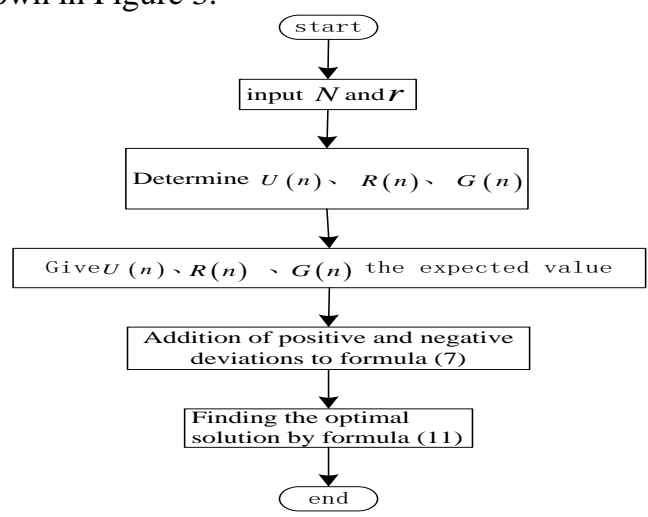

Figure 3: Flow chart of MMC optimal redundant configuration

Figure 3 includes the main steps of the MMC redundancy optimization configuration proposed in this article. The following will be verified by simulation.

\section{SIMULATION ANALYSIS}

In order to verify the feasibility and effectiveness of the redundant optimal configuration method for the MMC sub module proposed in this paper, a 5 level MMC simulation model is built in PSCAD. In this paper, the number of sub module failures is 1 , and a submodule of bridge arm in a phase occurs failure at $0.2 \mathrm{~S}$. At this time, the control signal of the module is blocked and the sub module is removed. The system MMC starts to run again at $0.21 \mathrm{~s}$. The waveforms of current and voltage in the simulation are shown in Figure 4.

Before failure occurs, MMC is in stable working condition, after failure of the fault module capacitor power storage, it is bound to cause the change of a energy, and $\mathrm{B}$ phase, $\mathrm{C}$ phase energy remained almost stable, which will cause the DC bus voltage rise, voltage of capacitor module a under the bridge arm of the A phase decreased slightly, the upper and lower bridge arm current asymmetry during the fault have certain fluctuations, as 
shown in Figure 4 (a), (b), (f) shown in. 0.01s continued to return to normal working condition. This is because the fault occurs, the control signal fault sub module is blocked, control bypass switch is closed, then switching to redundant sub modules, making it into the working state, the 5 sub-modules under the bridge arm has 3 sub modules, 2 sub modules in the work of the state, the working state of the swap, change the redundant state is in a normal working state, fault sub module for the conversion of redundant sub modules, as shown in Figure 4 (c), (d), which guarantees that each arm has 4 sub modules in the work of the state, 1 sub modules in the redundant state, so as to ensure the bridge arm voltage balance. Figure 4 (e) is a simulation waveform diagram of output current. From this diagram, it can be seen that there is little effect on output current after failure, which means that after adding redundant sub modules, the interference to the system is very small, and the reliability of the system is improved.

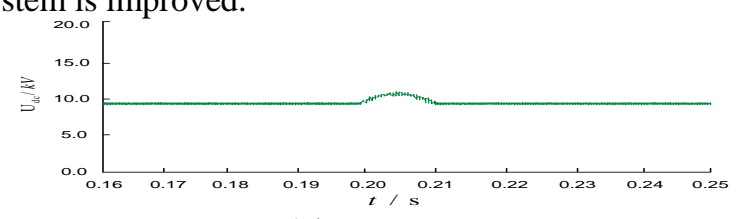

(a) DC Bus Voltage

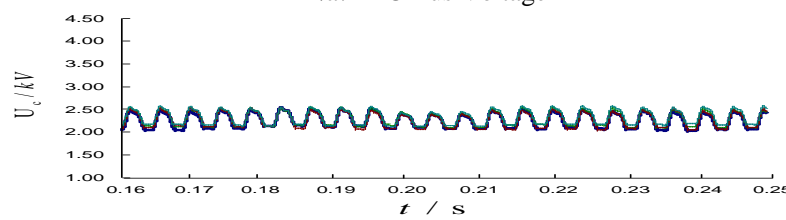

(b) Normal operating capacitance voltage of bridge arm under a phase

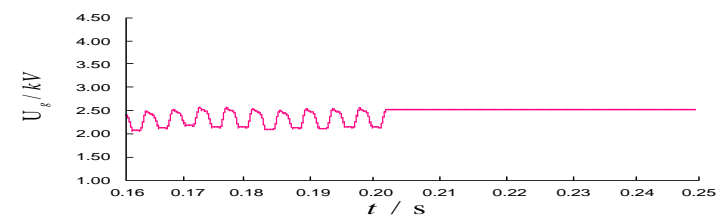

(c) Capacitance voltage in switching state of bridge arm under a phase

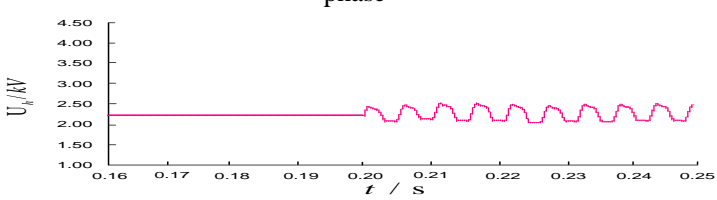

(d) Capacitance voltage of the submodule of bridge arm failure in a phase

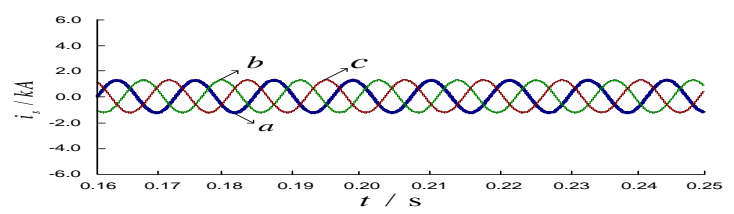

(e) Output current of three phase

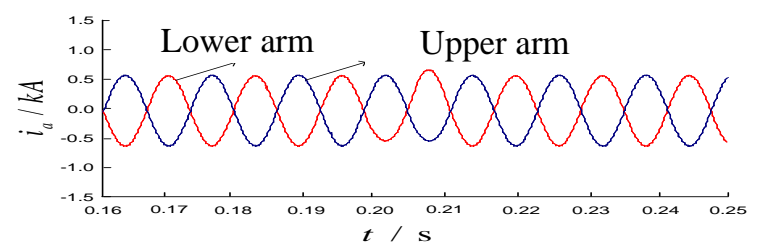

(f) The upper and lower bridge arm current of a

Figure 4: Simulative waveforms

\section{CONCLUSION}

In this paper, a reasonable and effective method for configuring redundant sub modules is proposed. Considering the 3 factors of redundant sub modules, such as the effective utilization of redundant modules, the number of redundant sub modules and the reliability of system MMC, a multi-objective optimization function with redundant configuration is established. Redundant configuration optimization scheme using the method of priority to solve in this paper, through the optimized value to accurately calculate the number of redundant configuration MMC module, based on the proposed optimization method, a 5 level MMC simulation model is built in PSCAD, the results verify the rationality and feasibility of the proposed optimization method.

\section{ACKNOWLEDGEMENTS}

Fund Project: National Key research program(2016YFB0900600)

\section{REFERENCES}

1. XU Bin, LI Chenghao, XIANG Wang, etc. MMC modular series parallel shunt expansion method and its application in energy Internet[J]. Power Construction, 2015, 36(10): 20-26.

2. LI Jiang, LI Guoqing. A survey on application of fault tolerant control in power system[J]. Power System Protection and Control, 2010, 38(3): 140-146.

3. WANG Chaoliang, ZHAO Chengyong, XU Jingzhong. A method for calculating sub-module redundancy configurations in modular multilevel converters[J]. Automation of Electric Power System, 2013, 37(16): 103-107.

4. Antonopoulos A, Angquist L, et al. Global asymptotic stability of modular multilevel converters[J]. IEEE Transactions on Industrial Electronics, 2014. 61(2): 603-612.

5. JING Huabing, NIAN Xiaohing. A novel protection strategy for sub-modules multilevel converter[J]. Power System Technology, 2013, 37(7): 1954-1958.

6. RAO Hong, SONG Qiang, LIU Wenhua, et al. Optimized design solutions for multi-terminal VSCHVDC system using modular multilevel converters and their comparison[J]. Power System Automation, 2013, 37 (15): 103-108.

7. LI Xiaoqian, LIU Wenhua, SONG Qiang, et al. An enhanced MMC topology with DC fault clearance capability [J]. Proceedings of the CSEE, 2014, 34(36): 6389-6397.

8. ZHOU Yuebin, JIANG Daozhuo, HU Pengfei, et al. A new approach for suppressing DC voltage ripples of MMC-HVDC[J]. Proceedings of the CSEE, 2013, 33(27): 36-43.

9. LI Tan, ZHAO Chengyong, LI luyao, et al. Submodule fault diagnosis and local protection scheme 
for MMC-HVDC system[J]. Proceedings of the CSEE, 2014, 34(10): 1641-1649.

10. LU Jingjing, LIU Zhengfu. MMC fault analysis and protection strategy for unified power quality controller[J]. Power Construction, 2015, 36(5): 3136. 